Supporting Information for

\title{
Gas Barrier Properties of Chemical Vapor-Deposited Graphene to Oxygen Imparted with Sub-eV Kinetic Energy
}

\author{
Shuichi Ogawa ${ }^{1, *}$, Hisato Yamaguchi ${ }^{2, \dagger}$, Edward F. Holby ${ }^{2}$, Takatoshi Yamada ${ }^{3, *}$, Akitaka \\ Yoshigoe $^{4}$, Yuji Takakuwa ${ }^{1}$ \\ ${ }^{1}$ Institute Multidisciplinary Research for Advanced Materials, Tohoku University, \\ Sendai 980-8577, Japan \\ ${ }^{2}$ Los Alamos National Laboratory, Los Alamos, New Mexico 87545, U.S.A. \\ ${ }^{3}$ National Institute of Advanced Industrial Science and Technology, Tsukuba, Ibaraki 305-8565, \\ Japan \\ ${ }^{4}$ Japan Atomic Energy Research Agency, Sayo, Hyogo 679-5148, Japan
}

*ogasyu@tohoku.ac.jp, †hyamaguchi@lanl.gov, †takatoshi-yamada@aist.go.jp

\section{Contents}

- Generation of supersonic molecular beams and its energy spread

- Preparation of specimen

- Raman spectroscopy of graphene

- Counter plots of Figure 1 (b) and (c)

- Conversion of $\mathrm{O}-\mathrm{Cu}$ peak intensities of $\mathrm{O} 1 \mathrm{~s}$ photoelectron spectra to adsorbed amount

- Comparison of $\mathrm{O}_{2}$ saturation rate in Figure 1 (h) with that of a bare substrate

- Second-order reaction model

- Separation of $\mathrm{C} 1 \mathrm{~s}$ photoelectron spectra prior to and after the irradiations

- $\mathrm{Cu} 3 p$ photoelectron spectra prior to and after the irradiations

- Molecular dynamics (MD) simulation details

- MD simulation results for pristine and large vacancy defect

- The adsorption probability increase as kinetic energy of the oxygen molecules increases for $\mathbf{E}_{\mathrm{t}}<0.83 \mathrm{eV}$ 


\section{Generation of supersonic molecular beams and its energy spread}

Figure S1 is a schematic for the generation of the supersonic molecular beam used in this study ${ }^{1}$. It begins with mixture of gases at thermal equilibrium in the nozzle. A trace amount of molecules of interest (oxygen in our study) are mixed into carrier gas(es) with different molecular weights to control their kinetic energy as details are described later in this section. Pressure of $>10^{-3}$ Torr is necessary to ensure thermal equilibrium of the gases, which follows the Maxwell-Boltzmann thermal distribution. Spraying the gases into higher vacuum of $10^{-5}$ Torr induces adiabatic expansion/free jet expansion. In this process, the thermal energies of the gases are converted to the kinetic energies directing to a wide range of angles. The critical component of the supersonic molecular beam generation in narrowing the energy spread of the beam is called a skimmer, which has a small physical opening with a diameter of about $0.1 \mathrm{~mm}$. This selects beams to gas molecules that are directing towards the specimen only in a narrow angle. Because the gas molecules directing in the same narrow angle possess kinetic energies with a narrow spread, energy spread that is $1-10 \%$ of that of thermal equilibrium molecular beam as shown in Figure S2 can be achieved by supersonic molecular beam ${ }^{1}$.

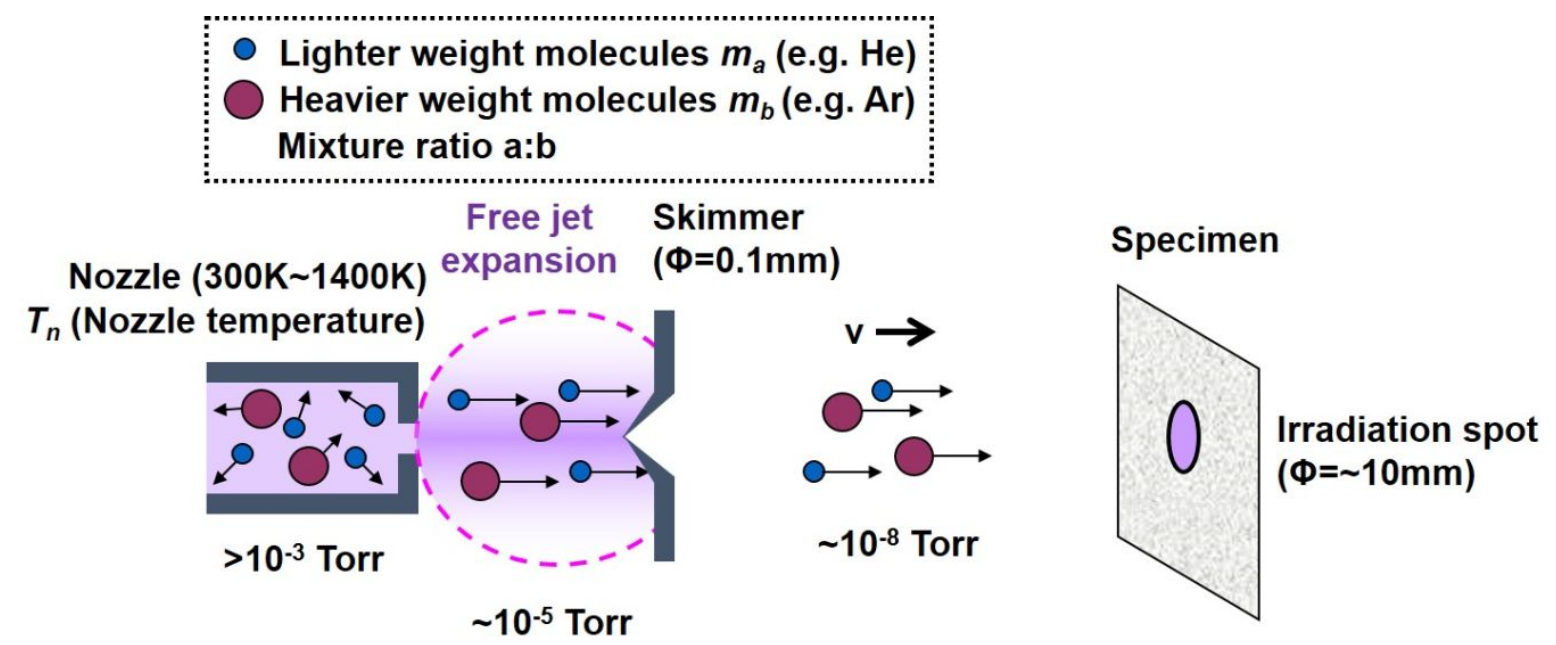

Figure S1. Schematic of supersonic molecular beam generation.
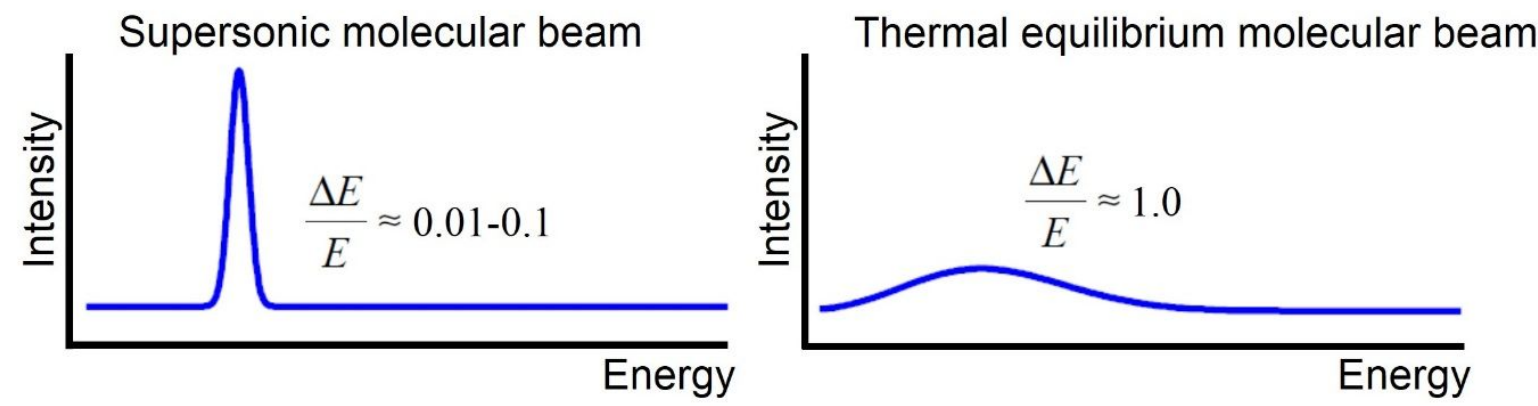

Figure S2. Schematic illustrating the stark difference in the energy spread of supersonic (left) and thermal equilibrium (right) molecular beam. 
Control of the molecular beam's kinetic energy can be performed by varying a nozzle temperature and/or the carrier gas ratio. When carrier gases of lighter weight with mass of $m_{a}$ and heavier weight with mass of $m_{b}$ are mixed by ratio a:b, $M$ below describes an average mass of gases in the nozzle. We used helium (He) and argon (Ar) gas for lighter and heavier weight carrier gases in our study, respectively.

$$
M=\frac{a m_{a}+b m_{b}}{a+b}
$$

The thermal energy is converted to kinetic energy via adiabatic expansion as follows.

$$
\frac{1}{2} m_{a} v^{2}=C_{p} T_{n} \frac{m_{a}}{M}
$$

where $v$ is velocity of the gas molecules, $C_{p}$ is the constant pressure specific heat, and $T_{n}$ is the nozzle temperature.

The equation indicates that kinetic energy of the molecular beam can be increased by increasing the ratio of lighter weight carrier gas and vice versa. Therefore, we used a higher ratio of He to generate supersonic molecular beams with higher kinetic energies, such as $E_{\mathrm{t}} \geq$ $0.83 \mathrm{eV}$, and we used a higher ratio of Ar to generate beams with lower energies, such as $E_{\mathrm{t}}$ below $0.83 \mathrm{eV}$. We kept the nozzle temperature to $1,400 \mathrm{~K}$ except for $E_{\mathrm{t}}=0.07 \mathrm{eV}$, which we used $300 \mathrm{~K}$ and the highest Ar ratio to obtain the lowest $E_{\mathrm{t}}$ possible with our system. Exhausting capability of our 1,000-L capacity turbo molecular pump (TMP) is the limiting factor in generation of the beam with lower energy.

\section{Preparation of specimen}

$\mathrm{Cu}(111)$ was deposited on a $c$-plane $\mathrm{Al}_{2} \mathrm{O}_{3}$ surface by radio frequency (RF) magnetron sputtering with a thickness of $3 \mu \mathrm{m}$. Theta- 2 theta pattern and a pole figure pattern of X-ray diffractions indicated a (111) orientation of $\mathrm{Cu}$ having a twin structure. Thermal CVD graphene was grown on this $\mathrm{Cu}(111)$ surface. The thermal CVD growth was performed in a rapid thermal annealing system with a base pressure of $10^{-5} \mathrm{~Pa}$. The substrate was first heated to $1,000{ }^{\circ} \mathrm{C}$ for $10 \mathrm{~min}$. in a $\mathrm{H}_{2} / \mathrm{Ar}(100 / 200 \mathrm{sccm})$ atmosphere at a process pressure of $40 \mathrm{~Pa}$ for a substrate cleaning. The graphene growth was then initiated by adding $\mathrm{CH}_{4}$ to the gas mixture with flow rates of $20 \mathrm{sccm}$. After 30 minutes of growth, the sample was cooled down in a $\mathrm{H}_{2} / \mathrm{Ar}$ atmosphere. Grown graphene is confirmed to be a high crystal quality monolayer based on Raman spectroscopy. 


\section{Raman spectroscopy of graphene}

Raman spectroscopy was performed on our as-grown graphene on $\mathrm{Cu}(111)$ substrate using a $488 \mathrm{~nm}$ laser. Use of graphene without transfer allows us to investigate a specimen that is prepared in the identical procedures as that of our study. The 488-nm laser eliminates a broad background signal from the $\mathrm{Cu}$ substrates that are often observed for a case of $532 \mathrm{~nm}$. Obtained spectra as in Figure S3 for our graphene was consistent with that of high crystal quality mechanically-cleaved monolayer graphene. Specifically, absence of a D peak around $1,350 \mathrm{~cm}^{-1}$ indicates that there are no significant defects ${ }^{2}$. A sharp single Lorentzian peak of 2D peak around 2,700 $\mathrm{cm}^{-1}$ and roughly 4 times stronger intensity of $2 \mathrm{D}$ peak over $\mathrm{G}$ peak around $1,580 \mathrm{~cm}^{-1}$ indicate that our graphene is monolayer. ${ }^{2} \mathrm{FWHM}$ of $28.0 \mathrm{~cm}^{-1}$ for $2 \mathrm{D}$ peak, which is comparable to $\sim 25 \mathrm{~cm}^{-1}$ of mechanically-cleaved graphene, further indicates that our graphene is of high crystal quality ${ }^{2}$.

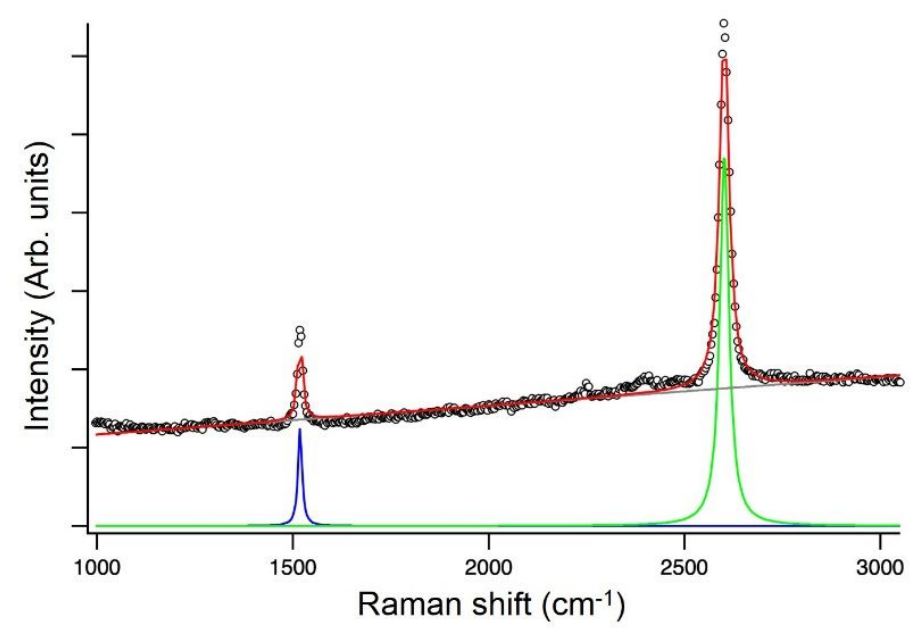

Figure S3. A typical Raman spectrum of our graphene taken at $488 \mathrm{~nm}$ (black open circles). Intensity is in arbitrary units. G peak around $1,580 \mathrm{~cm}^{-1}$ (blue solid line) and 2D peak around $2,700 \mathrm{~cm}^{-1}$ (light green solid line) are fitted using the Lorentzian function after background subtraction (light brown solid line). 
(a)

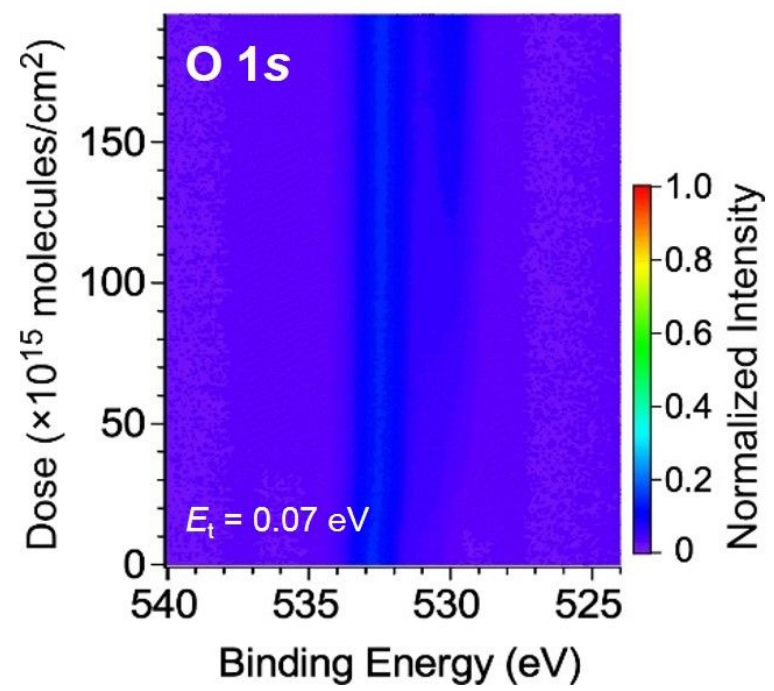

(b)

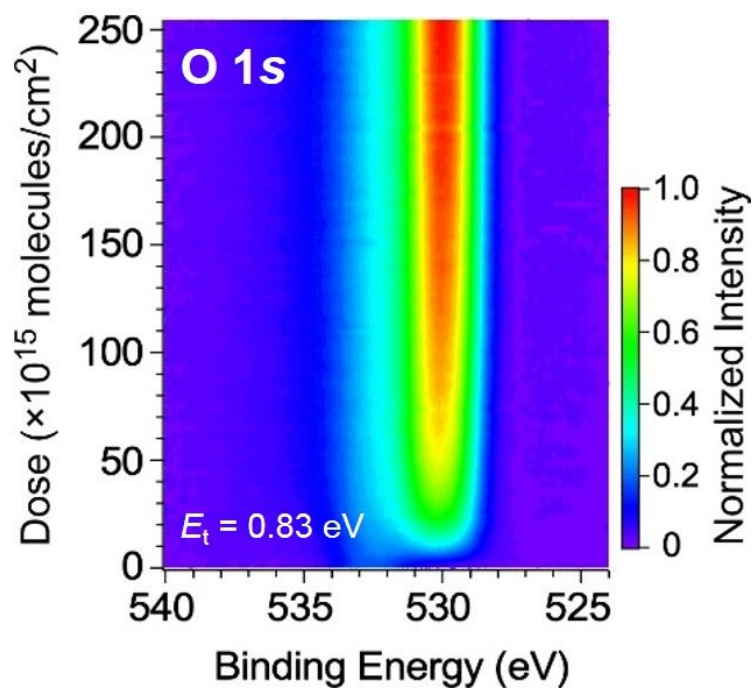

Figure S4. Counter plots of (a) Figure 1 (b) and (b) Figure 1 (c), respectively.

Conversion of $\mathrm{O}-\mathrm{Cu}$ peak intensities of $\mathrm{O} 1 \mathrm{~s}$ photoelectron spectra to adsorbed amount

Copper has face-centered cubic (fcc) crystal structure with a lattice constant of $a=3.61496$

$\AA$, thus the atom density of 1 monolayer ( $1 \mathrm{ML}$ ) for the $\mathrm{Cu}(111)$ surface is $1.7672 \times 10^{15}$ atoms $/ \mathrm{cm}^{2}$. 0.4 ML is the theoretical full coverage of oxygen atoms on the $\mathrm{Cu}(111)$ surface ${ }^{3}$. In converting our $\mathrm{O}-\mathrm{Cu}$ peak intensities of $\mathrm{O} 1 s$ photoelectron spectra to ML coverage on $\mathrm{Cu}(111)$, our procedures were as follows. We first fitted our molecular oxygen dose dependence of the $\mathrm{O}-\mathrm{Cu}$ peak intensity for $E_{\mathrm{t}}=0.83 \mathrm{eV}$ with the second-order reaction model (Figure S6). We then converted the saturated value of the fit (indicated by the black arrow of Figure S5) to $0.4 \mathrm{ML}$. Validity of our conversion relies on the fact that all of molecular oxygen dose dependence of the $\mathrm{O}-\mathrm{Cu}$ peak intensity saturated at similar values for $E_{\mathrm{t}} \geq 0.83 \mathrm{eV}$, which is indicative of full coverage of oxygen on $\mathrm{Cu}(111)$.

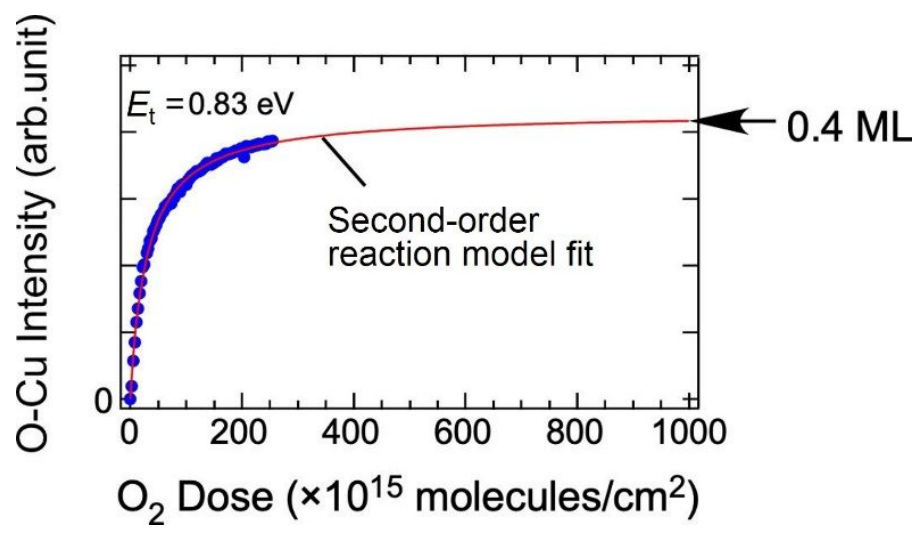


Figure S5. Molecular oxygen dose dependence of the $\mathrm{O}-\mathrm{Cu}$ intensity for $E_{\mathrm{t}}=0.83 \mathrm{eV}$ (filled blue circle) fitted by the second-order reaction. Black arrow at the saturated intensity around $\mathrm{O}_{2}$ dose of $1,000 \times 10^{15}$ molecules $/ \mathrm{cm}^{2}$ (solid red line) is converted to 0.4 ML.

We have also tried fitting our data using the first-order reaction model (so called "Langmuir adsorption model"), which assumes one type of adsorbate instead of two ${ }^{4}$. The fit was good for the lower dose but mismatch became larger as the dose increased. Determining details of the adsorption mechanism of molecular oxygen onto the $\mathrm{Cu}(111)$ surface is out of the scope of this study. However, what is critical for our case is that there is an established model that fits our data well and that we have fitted all of our data with the same model to draw a conclusion regarding the comparison between adsorption probabilities of molecular oxygen onto $\mathrm{Cu}(111)$ depending on $E_{\mathrm{t}}$.

\section{Second-order reaction model}

The second-order reaction model can be used to explain time dependent adsorbates coverage/thin film growth on a surface of a solid ${ }^{3}$. It describes a solid surface in one dimension as in its sideview and assumes two types of adsorbates, which can be the same. Figure S6 is a schematic of the model. The pink region at the bottom indicates a solid surface and $\theta$ indicates a region with adsorbates, thus 1- $\theta$ indicates a region without adsorbates.

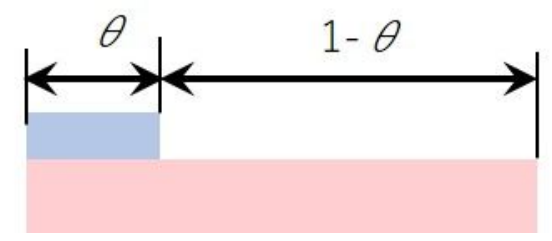

Figure S6. Schematic of a solid surface (pink) with adsorbates (light blue). The model considers one dimension as in its sideview. $\theta$ and $1-\theta$ indicate regions with and without adsorbates, respectively.

The model assumes that adsorbates react preferentially where there is no adsorbate. Therefore, coverage area of adsorbates would have a linear dependence on their adsorption probabilities. We assume two adsorbates to be the same, thus time-dependent adsorbate coverage on the surface can be expressed as below. $K$ is the absorbing probability of our absorbate.

$$
\frac{d \theta}{d t}=K^{2}(1-\theta)^{2}
$$

By replacing $K^{2}$ with $k$, the equation can be rewritten as

$$
\frac{d \theta}{d t}=k(1-\theta)^{2} \rightarrow \theta=A\left(1-\frac{1}{1+k t}\right)
$$


where $A$ is the saturated amount.

This is the equation that we used to fit our experimental data.

\section{Comparison of $\mathrm{O}_{2}$ saturation rate in Figure $1(\mathrm{~h})$ with that of a bare substrate}

Our $\mathrm{O}_{2}$ saturation rate for $E_{\mathrm{t}} \geq 0.83 \mathrm{eV}$ in Figure $1(\mathrm{~h})$ matched very well with that of a graphene-free bare $\mathrm{Cu}(111)$ substrate for oxygen molecular beam exposure with $E_{\mathrm{t}}=0.6 \mathrm{eV}^{3}$. Slight deviation at the low dose region $\left(<100\right.$ molecules $\left./ \mathrm{cm}^{2} / \mathrm{s}\right)$ is most probably due to difference in the measurement methods. Our measurements were performed simultaneously (i.e. in situ) while the data in literature were performed separately (i.e. repeat of oxygen molecular beam exposure followed by XPS).

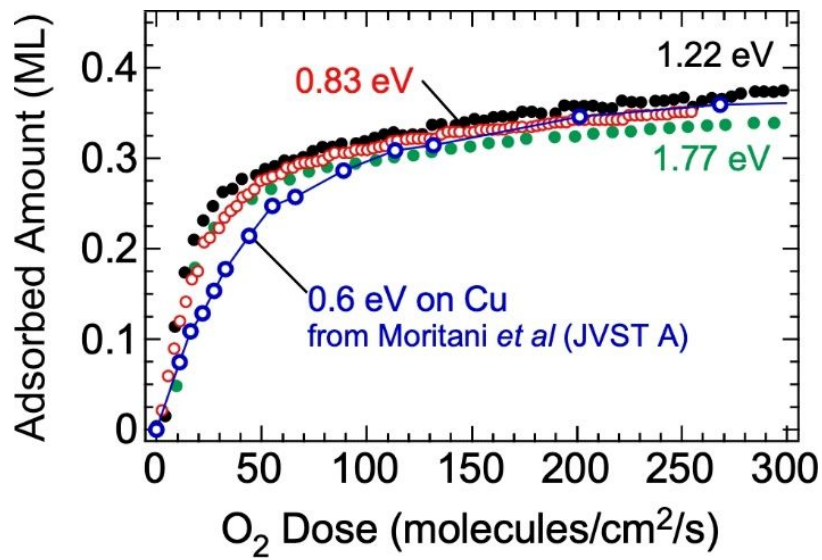

Figure S7. Comparison of $\mathrm{O}_{2}$ saturation rate in Figure 1 (h) with that of a graphene-free bare $\mathrm{Cu}(111)$ substrate $^{3}$. Data point of $(0,0)$ is added for the bare substrate as an eye guide.

\section{Separation of $\mathrm{C} 1 \mathrm{~s}$ photoelectron spectra prior to and after the irradiations}

Figure 2(b) compares $C 1 s$ photoelectron spectra for $E_{\mathrm{t}}=0.07$ and $1.77 \mathrm{eV}$ prior to and after irradiation without peak separations. Figure S8 shows those with peak separations. There is no peak corresponding to vacancy defects at $-0.3 \mathrm{eV}$ of the $s p^{2}$ peak in any of the spectra ${ }^{5}$.
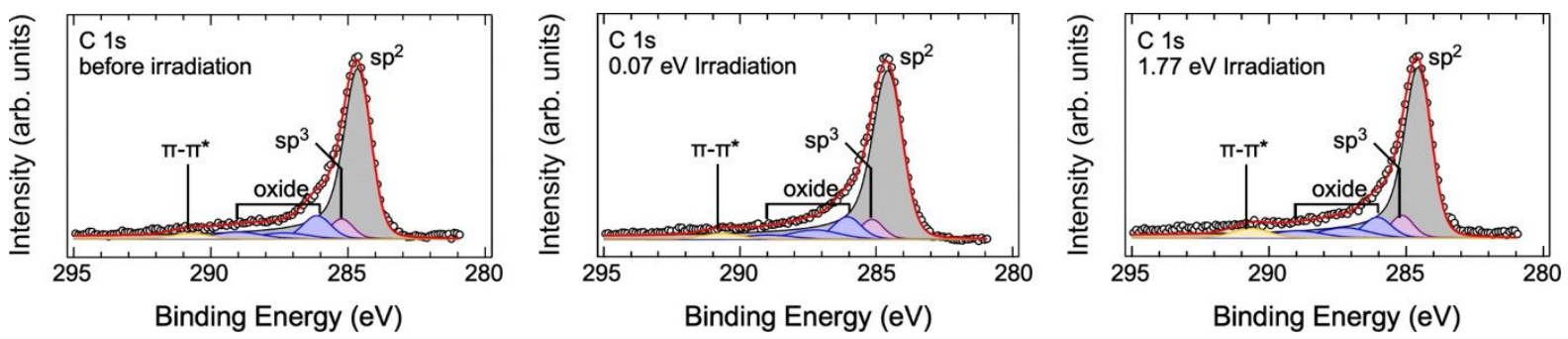

Figure S8. Separated peaks for Figure 2(b). 


\section{$\mathrm{Cu} 3 p$ photoelectron spectra prior to and after the irradiations}

$\mathrm{Cu} 3 p$ photoelectron spectra before and after the oxygen beam irradiation with $E_{\mathrm{t}}=1.77 \mathrm{eV}$.

The spectrum for after the irradiation clearly shows a presence of non-oxidized copper, indicating that the oxidized region is limited such as to the very top surface (i.e., monolayer).

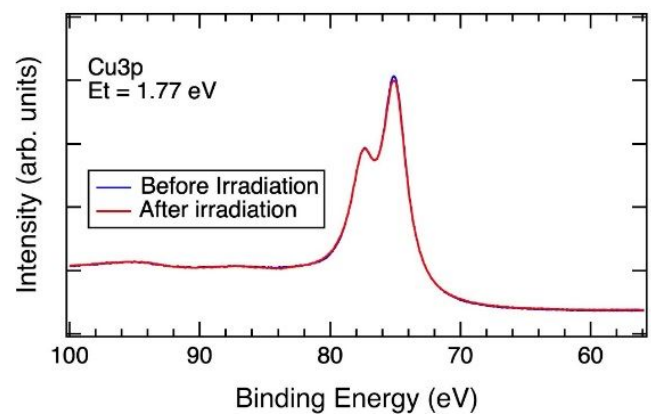

Figure S9. Cu $3 p$ photoelectron spectra of before (blue) and after (red) the oxygen beam irradiation with $E_{\mathrm{t}}=1.77 \mathrm{eV}$.

\section{Molecular dynamics (MD) simulation details}

ReaxFF 6 , a parameterized force field describing the interaction of atomic species that allows for bond making/breaking, was used to study how molecular $\mathrm{O}_{2}$ interacts with a 6-layer (underlayers not shown in Figure 3 for clarity) graphite $6 \times 6$ periodic slab with defects in the first interaction layer using molecular dynamics approaches. 400-fs simulations were completed with 0.02-fs time steps within the NVE "molecule gun" approach within the Amsterdam Density Functional Suite (ADF). The dispersion/CHONSSi-lg.ff: (C/H/O/N/S/Si) force field of Liu et al. ${ }^{7}$ was used for describing the system Hamiltonian as it contains required $\mathrm{C}$ and $\mathrm{O}$ species in the training set along with treating dispersion forces and enabling consideration of bond making/breaking, unlike classical interatomic potentials. Similar results were found with different force fields as well. In numerous previous examples, ReaxFF has been shown to capture relevant thermokinetic energies and reaction pathways in oxygencarbon systems ${ }^{8}$.

\section{MD simulation results for pristine and large vacancy defect}

MD simulation movies are also available as supplementary information. Figure S10 demonstrates the results for two cases of (1) molecular oxygen with $1.5 \mathrm{eV}$ approaching a pristine surface (left) and (2) molecular oxygen with $0.5 \mathrm{eV}$ approaching the large vacancy defect surface mentioned in the main text (right). The $1.5 \mathrm{eV}$ oxygen bounces back and the $0.5 \mathrm{eV}$ oxygen permeates through, highlighting the uniqueness of the oxygen molecules with 1.0 and $1.5 \mathrm{eV}$ permeating through divacancy defected graphene as described in the main text. 


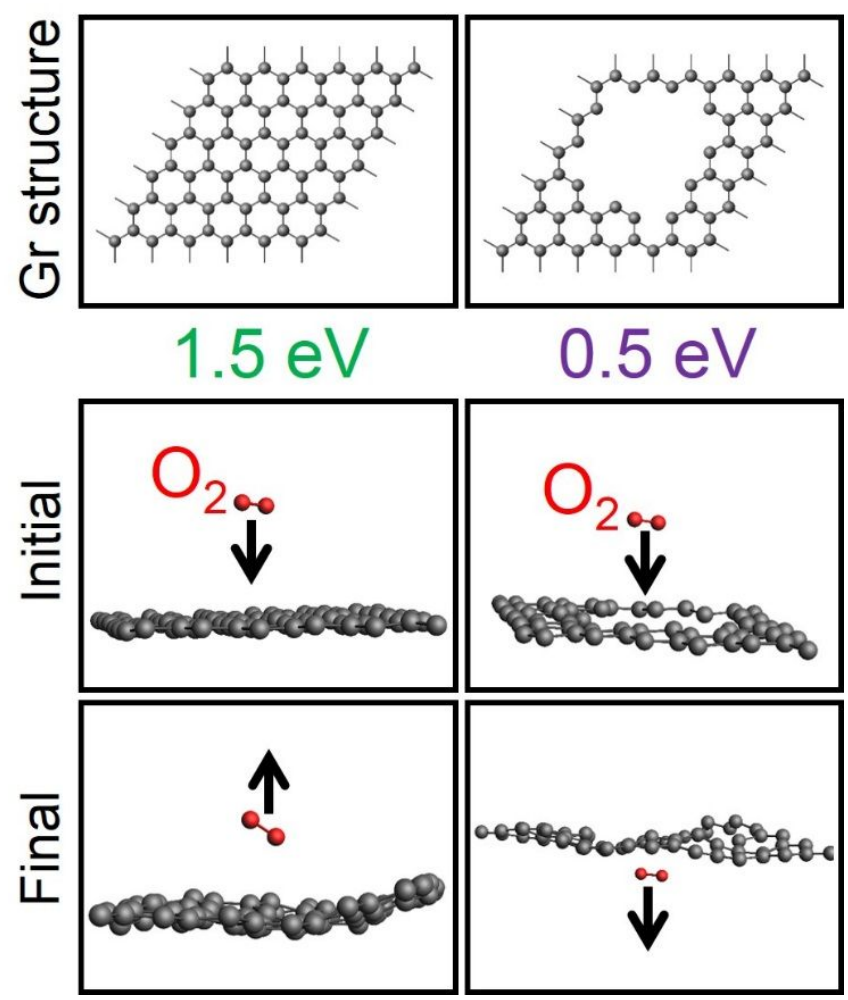

Figure S10. MD simulation results for molecular oxygen with $1.5 \mathrm{eV}$ approaching a pristine surface (left) and molecular oxygen with $0.5 \mathrm{eV}$ approaching the large vacancy defect surface mentioned in the main text (right). Red and gray filled circles indicate oxygen and carbon atoms, respectively.

\section{The adsorption probability increase as kinetic energy of the oxygen molecules increases} for $\mathrm{E}_{\mathrm{t}}<\mathbf{0 . 8 3 e V}$

Figure S11(a) is the kinetic energy-dependent adsorption probability extracted from Figure 1(h). The $y$-axis is on a log scale. An exponential-like increase of the adsorption probability as the kinetic energy increases for $E_{\mathrm{t}}<0.83 \mathrm{eV}$ resembles that of the bare $\mathrm{Si}$ case ${ }^{9-11}$, and it indicates the adsorption is not a physisorption-driven process. An adsorption will decrease as the kinetic energy increases, if it were the case. We performed additional MD simulations to gain insights into the origin of this behavior, which the results indicated to be the presence of vacancy defects larger than divacancy. Figure S11(b) is the summary of results for all MD simulations performed in this study. Specifically, oxygen molecules with kinetic energy of $0.5 \mathrm{eV}$ dissociated at the defect sites with 3-and 5-atom vacancies. This is in sharp contrast to the case of divacancy (Figure 3), which required kinetic energies of $>1.0 \mathrm{eV}$ for the dissociation to occur. The results indicate there is a decrease in kinetic energy required for oxygen molecules to dissociate and bond to copper substrates as the number of carbon vacancy atoms increases. This is consistent with the extreme case of defect-free graphene, where the oxygen molecules of all kinetic energies $(0.5,1.0$, and $1.5 \mathrm{eV})$ bounced back. It is known that defect sizes of 3 and 
5 atoms do exist in CVD graphene ${ }^{12}$, even for those without a D-peak by Raman spectroscopy, as in our case. This could explain the increase of adsorption probability as kinetic energy increases for $E_{\mathrm{t}}<0.83 \mathrm{eV}$ in Figure 1(h). Our MD simulation results also indicate that as the defect size increases, there is a crossover from a catalyzed dissociation mechanism to ballistic transport of $\mathrm{O}_{2}$ to the underlayer, which seems to be around 7-atom vacancy. The 7-atom vacancy was an intermediate state in that an oxygen molecule dissociates in some cases and in other cases transports through graphene without dissociation, although it interacts with vacancy-edge carbon atoms. Movies of typical simulation results are available as a part of supporting information.

(a)

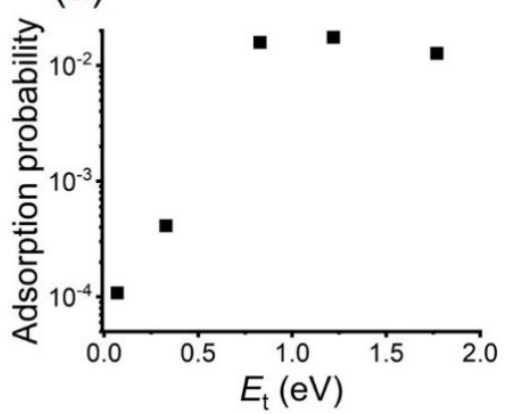

(b)

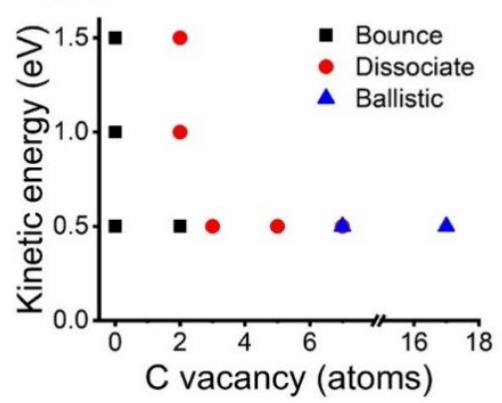

Figure S11. (a) Kinetic energy-dependent adsorption probability extracted from Figure 1(h). $\mathrm{Y}$-axis is on a log scale. (b) Summary of MD simulation results for an oxygen molecule approaching $0,2,3,5,7$, and 17 atom vacancy defects in graphene surfaces. "Bounce" indicates the oxygen molecules bounced back, "Dissociate" indicates the molecules dissociated and bonded to the vacancy-edge carbon atoms, and "Ballistic" indicates the molecules transported through the vacancies ballistically or with some interactions with vacancy-edge carbon atoms without dissociation of the molecule or bonding to the vacancy-edge carbon atoms.

\section{References}

1. Parker, H. M., Kuhlthau, A. R., Zapata, R. Rarefield Gas Dynamics. Pergamon Press Inc.: N.Y., 1960.

2. $\quad$ Ferrari, A. C.; Meyer, J. C.; Scardaci, V.; Casiraghi, C.; Lazzeri, M.; Mauri, F.; Piscanec, S.; Jiang, D.; Novoselov, K. S.; Roth, S.; Geim, A. K. Raman Spectrum of Graphene and Graphene Layers. Physical Review Letters 2006, 97 (18), 187401.

3. Moritani, K.; Okada, M.; Sato, S.; Goto, S.; Kasai, T.; Yoshigoe, A.; Teraoka, Y. Photoemission study of the translational energy induced oxidation processes on $\mathrm{Cu}(111)$. Journal of Vacuum Science \& Technology A 2004, 22 (4), 1625-1630.

4. Tang, J. Y.; Nishimoto, K.; Ogawa, S.; Takakuwa, Y. Strong Temperature Dependence of the Initial Oxide Growth on the Si(111) 7 x 7 Surface. e-Journal of Surface Science and Nanotechnology 2012, 10, 525-529. 
5. Ogawa, S.; Yamada, T.; Ishidzuka, S.; Yoshigoe, A.; Hasegawa, M.; Teraoka, Y.; Takakuwa, Y. Graphene Growth and Carbon Diffusion Process during Vacuum Heating on $\mathrm{Cu}(111) / \mathrm{Al}_{2} \mathrm{O}_{3}$ Substrates. Japanese Journal of Applied Physics 2013, 52 (11R), 110122. 6. $\quad$ Senftle, T. P.; Hong, S.; Islam, M. M.; Kylasa, S. B.; Zheng, Y.; Shin, Y. K.; Junkermeier, C.; Engel-Herbert, R.; Janik, M. J.; Aktulga, H. M.; Verstraelen, T.; Grama, A.; van Duin, A. C. T. The ReaxFF reactive force-field: development, applications and future directions. npj Computational Materials 2016, 2 (1), 15011.

7. Liu, L.; Liu, Y.; Zybin, S. V.; Sun, H.; Goddard, W. A. ReaxFF-lg: Correction of the ReaxFF Reactive Force Field for London Dispersion, with Applications to the Equations of State for Energetic Materials. The Journal of Physical Chemistry A 2011, 115 (40), 1101611022 .

8. Bagri, A.; Mattevi, C.; Acik, M.; Chabal, Y. J.; Chhowalla, M.; Shenoy, V. B. Structural evolution during the reduction of chemically derived graphene oxide. Nat Chem 2010, 2 (7), 581-587.

9. Yoshigoe, A.; Teraoka, Y. Adsorption Dynamics on Si(111)-7 × 7 Surface Induced by Supersonic $\mathrm{O}_{2}$ Beam Studied Using Real-Time Photoelectron Spectroscopy. The Journal of Physical Chemistry C 2010, 114 (51), 22539-22545.

10. Teraoka, Y.; Yoshigoe, A. Precise Control of Si(001) Initial Oxidation by Translational Kinetic Energy of $\mathrm{O}_{2}$ Molecules. Japanese Journal of Applied Physics 2002, 41 (Part 1, No. 6B), 4253-4260.

11. Teraoka, Y.; Yoshigoe, A. Erratum: "Precise Control of Si(001) Initial Oxidation by Translational Kinetic Energy of $\mathrm{O}_{2}$ Molecules”. Japanese Journal of Applied Physics 2015, 54 (3), 039204.

12. Huang, S.; Dakhchoune, M.; Luo, W.; Oveisi, E.; He, G.; Rezaei, M.; Zhao, J.; Alexander, D. T. L.; Züttel, A.; Strano, M. S.; Agrawal, K. V. Single-layer graphene membranes by crack-free transfer for gas mixture separation. Nature Communications 2018, $9(1), 2632$. 